\title{
Design and Performance Analysis of Adaptive Discriminator
}

\author{
Sanjay Kumar Sharma \\ ${ }^{1}$ Department of Electronics and Communication Engineering, University Institute of Technology \\ Rajiv Gandhi Proudyogiki Vishwavidyalaya (RGPV), Bhopal India
}

\begin{abstract}
Nuclear and High Energy Physics experiments often deal with a large number of detectors. These detectors give out analog signals depicting several parameters like time of arrival, amplitude and width of these analog signals, and pulse count rate etc. Usually, fast discriminators are used to convert these analog signals to digital form, crossing a set threshold. We have designed and developed 8 channel leading edge fast adaptive discriminator module with independent programmable threshold. This module is developed on a CAMAC (IEEE 583) standard for programmability. We discuss the design aspects as well as the performance of a proto-type module in this paper.
\end{abstract}

Keywords: ADC, Max1311, Analog Multiplexer, ADG526A, Read out, Adaptive, CAMAC, DAC, Max547, Comparator, Max9600, High Energy Physics, NIM

\section{Introduction}

High Energy and Nuclear Physics experiments often deal with a large number of information channels which produce analog signals. In these experiments, some or all of these analog signals have to be used to validate the final event as well as to extract different parameters before recording. Usually, all these analog signals crossing a set threshold are converted to digital signal using fast discriminator.

The threshold of these discriminators are changed online or offline as per the need of experiment. Many commercial discriminator modules are available with manual threshold adjustments or programmable common threshold adjustment. Many times it is necessary to read out the set thresholds for various calculations and in many cases it is essential to monitor these set thresholds. Whenever it is necessary, one has to adjust these thresholds online or offline as per the demand of the data is being recorded.

Many times it is essential to adjust, control, and monitor the threshold of each channel individually as done, for example, in experiments searching for sources of high energy celestial $\gamma$-rays using atmospheric Cherenkov technique.

The Cherenkov light pool produced by primary $\boldsymbol{\gamma}$-rays or cosmic-rays in the atmosphere lasts for a few ns. It is sampled during moon less dark nights by an array of Atmospheric Cerenkov telescopes in these ground-based experiments. Photomultiplier tube (PMT), mounted at the focus of the light collector, and converts the light to electrical signal. The shape of the PMT pulse depends on the density and the arrival time distribution of Cherenkov photons and on the response of the detector elements $[1][2][3][4]$

Due to large collection area, there is a spread in the arrival time of photon particles, which causes fluctuations in the shape of the output analog pulse of the radiation detector. These experiments have a large number of fast analog channels of information which have to be first digitalized using discriminator for fast trigger validation, data monitoring as well as recording.

The efficiency of each channel, which consists of MirrorPMT, has to be maintained uniformly throughout the observation time / duration of a given experiment. This could be achieved by using discriminator which has features of programmable threshold, remote adjustment and monitoring.

Thus there was a need for independent remote adjustment $\&$ monitoring of several discriminator channels along with readout and adaptive features. These readout and adaptive features maintain the threshold value to keep the gain of each channel constant. Keeping these important needs, of nuclear and high energy physics experiments, in mind, we have designed a general purpose 8 channel adaptive discriminator module for using in the existing Computer Automated Measurement and Control CAMAC [IEEE 583] standard based data acquisition system ${ }^{[5][6][7][8]}$.

Proposed design of 8 channel leading edge adaptive discriminator has independent programmable threshold of range 0 to $-2.0 \mathrm{~V}$ with readout and adaptive features. We discuss here the design features of this module including the circuit diagram and its performance.

\section{Basic Principle of Leading Edge Discriminator}

Detectors or transducers used in high energy \& nuclear physics experiments generate analog pulses of varying amplitude and shape; these pulses are random in nature. The discriminators are generally used to convert these analog pulses to more practical digital world. Discriminator produces a standard pulse which is related in time to the leading edge crossing of the threshold ${ }^{[9][10]}$. In a leading edge discriminator, the timing of the digital pulse varies with the amplitude of the input signal due to a fixed threshold. The variation in arrival time of the output pulse with varying amplitude of the input signal of same rise time is called discriminator 'time-walk ${ }^{17][9]}$ 


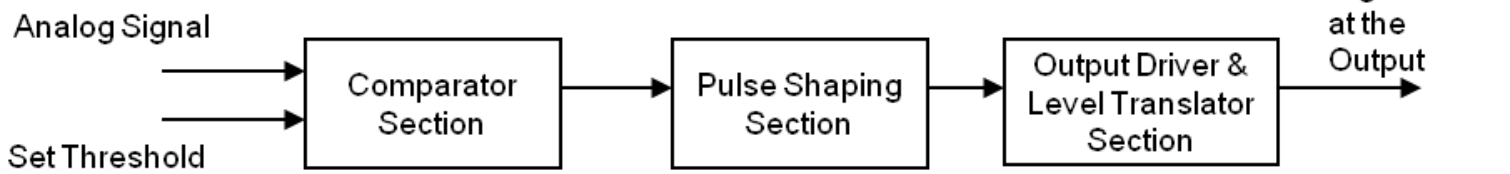

Figure 1: Block diagram of leading edge discriminator

Generally, a discriminator is built around sub-modules as shown in Fig. 1. The comparator section in the first stage detects the leading and trailing edge of input signal crossing the set threshold as a boundary for the width of output pulse having constant pulse height.

The pulse shaping section reshapes the pulse to a desired width preserving arrival time information of analog pulse which is in time correlation with comparator leading edge.

Finally, the driver cum translator section would convert the shaped digital signals to user desired output standard for driving long cables. During complete process of conversion the arrival time information of analog signals is preserved.

\section{Leading Edge Discriminator Channel Design}

In proposed design the leading edge discriminator is built with the requirements of variable threshold, fixed width, threshold readout, and real time monitoring of the required threshold. We have chosen a Maxim make Dual Channel Voltage Comparator with ECL outputs, Max $9600^{[11]}$ in our design as a leading edge comparator.

The comparator is configured for negative polarity input pulses of range 0 to $-2.0 \mathrm{~V}$ and without Latch enable functions (by keeping LE at low and LE_ at high level), it is also configured to zero hysteresis by connecting $\mathrm{HYS}_{\mathrm{A}}$ to Vcc. Clamping diodes are used for input voltage protection.
Motorola make mono-shot chip, MC $10198^{[12]}$, is used for shaping the pulse width to a fixed value of $20 \mathrm{~ns}$. This mono-shot is triggered by leading edge of comparator and is operated in non-retriggerable mode. Transistor based ECL (Emitter Coupled Logic) to NIM (Nuclear Instrumentation Module) translator is incorporated at the output side.

The NIM output can drive up to $50 \Omega$ load. The arrival time information of analog signals is preserved till final output while processing through all sub-modules.

\section{Eight Channel Adaptive Discriminator}

The module we have designed is an eight channel leading edge adaptive discriminator with independent threshold control. It is designed using CAMAC standard for programmability of threshold and it can be varied from 0 to $2 \mathrm{~V}$ with a 12-bit resolution. The overall functionality of the 8 channel programmable adaptive discriminator is depicted in Fig. 2.

The threshold of each channel is controlled by a DAC output by using Octal 13 bit bipolar DAC chip called MAX547 $7^{[1]}$. The DAC is interfaced with CAMAC bus for user programmability of individual DAC output. For reading back the set threshold, we have used ADC chip MAX1311 $1^{[11]}$ from Maxim Integrated Products and an analog multiplexer ADG526 $\mathrm{A}^{[13]}$ with 12 bit resolution. The LEMO connectors mounted on the front panel are used for connecting all analog inputs and the corresponding discriminator outputs.

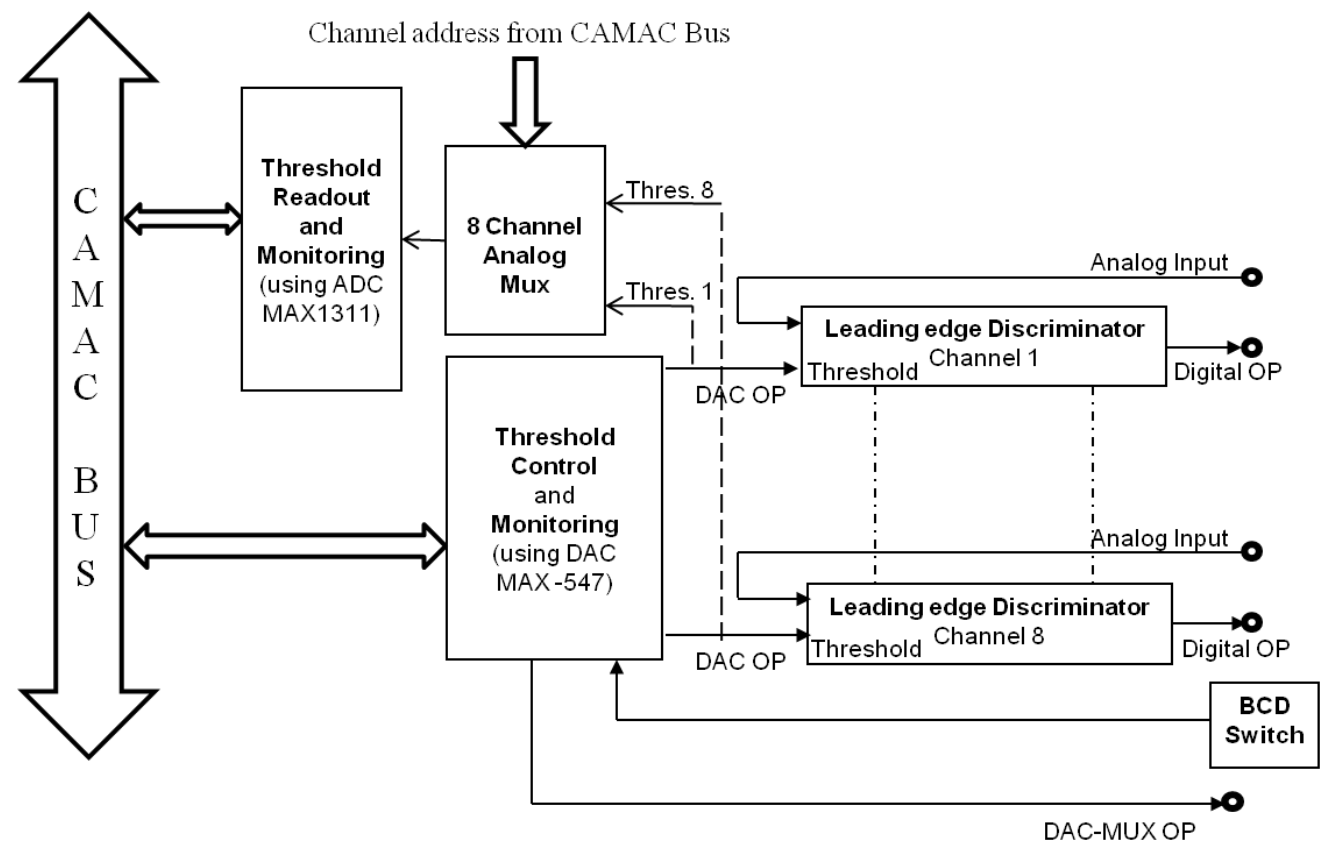

Figure 2: Block diagram of eight channel programmable adaptive discriminator 


\section{International Journal of Science and Research (IJSR) \\ ISSN (Online): 2319-7064}

Index Copernicus Value (2013): 6.14 | Impact Factor (2015): 6.391

\subsection{Threshold Control}

This section mainly controls the thresholds for individual discriminator channel over a range of 0 to $-2 \mathrm{~V}$ with a 12-bit resolution. It comprises of mainly three sub-units called CAMAC interface, Octal DAC unit and Multiplexer. The CAMAC interface decodes the CAMAC commands issued by user program and loads the threshold number to the input latch of the addressed DAC.

These latched input values are loaded to all the DACs simultaneously by a user CAMAC command. The DAC output voltage is given by the formula $\mathbf{V}_{\text {out }}=\mathbf{V}_{\text {ref }} *$ [(D/4096) -1]. Where, D is the 13-bit binary numeric value of the DAC input and it ranges from 0 to 8191.

The resolution is computed as $\mathbf{V}_{\text {ref }}$ / 4096. In our design, we have tied all 4 reference inputs $\left(\mathrm{V}_{\text {ref }}\right)$ together and it is set at $2 \mathrm{~V}$ using tunable precise voltage source. We demand $\mathrm{D}$ to be within 4095 as we deal with only negative polarity pulses and hence threshold can be varied between 0 to $-2 \mathrm{~V}$ with a resolution of $0.5 \mathrm{mV}$. The resolution can be changed by changing the reference voltage as per the requirement of the experiment.

\subsection{Threshold read out}

This section mainly readouts the set thresholds for individual discriminator channel over a range of +5 to $-5 \mathrm{~V}$ with a $12-$ bit resolution. It comprises of mainly three sub-units called CAMAC interface, ADC unit and an analog multiplexer.

The CAMAC interface decodes the CAMAC commands issued by user program and reads the threshold value of the addressed ADC. The ADC gives 12 bit 2 es complement digital output data code. The resolution is $\mathbf{1} \mathbf{L S B}=\mathbf{4} \mathbf{X} \mathbf{V}_{\text {ref }}$ $/ \mathbf{2}^{\mathbf{1 2}}$. In our design, we have used internal 2.5 volt as a reference inputs $\left(\mathrm{V}_{\text {ref }}\right)$ with a resolution of $2.44 \mathrm{mV}$.

\section{Real Time Threshold Monitoring and Adjustment}

The set threshold is read back via CAMAC read bus through read back section which comprises of $\mathrm{AMUX}^{[13]}$ and $\mathrm{ADC}^{[11]}$ and these read threshold values are compared with the adjusted / set thresholds. If the difference between these two threshold values is more than the set / reference limit and at the same time all other parameters i.e. supply voltages, sky condition, high voltage supply etc. are constant and are well within the set limits then the end user program automatically starts varying the set threshold for the module till it is not reaching the set count rate.

\section{CAMAC commands for the adaptive discriminator functioning}

The format of a typical CAMAC ${ }^{[5][6][7]}$ command is N,A,F, D24; where D24 is 24 bit Write data or it returns 24 bit Read data. Where $\mathrm{N}$ is Station address, $\mathrm{A}$ is Station subaddress, $\mathrm{F}$ is the function. The valid commands for adaptive discriminator module are listed below:

- $\quad \mathrm{N}, \mathrm{A}(0$ to 7$), \mathrm{F}(16), 13$ bit data command writes 13 bit data to input latch of $\overline{D A C}$ selected by sub address $\mathrm{A}^{-}$.

- $\quad \mathrm{N}, \mathrm{A}(\mathrm{x}), \mathrm{F}(8)$, data(x) command loads data in the input latches to all DACs simultaneously.

- $\quad \mathrm{N}, \mathrm{A}(\mathrm{x}), \mathrm{F}(9)$, data(x) or $\mathrm{C}$ or $\mathrm{Z}$ command initializes all DACs to zero value. Where, $x$ denotes Don't care.

- $\quad \mathrm{N}, \mathrm{A}(\mathrm{x}), \mathrm{F}(10)$, or $\mathrm{C}$ or $\mathrm{Z}$ command initializes / clears both the address and enable data in the latches resulting in no output (all switches off) in AMUX ADG526A.

- $\mathrm{N}, \mathrm{A}(0$ to 2$), \mathrm{F}(17)$ latches the state of the address control lines and the enable line.

- $\quad \mathrm{N}, \mathrm{F}(7)$, and strobe signal S2 reads 12 bit data.

\section{Calibrations}

The reference voltages for all the DACs, set at $-2.0 \mathrm{~V}$ and for ADC $2.5 \mathrm{~V}$, and both the reference voltages are found to be stable with and load and time as shown in Fig. 3a and Fig. 3b respectively.

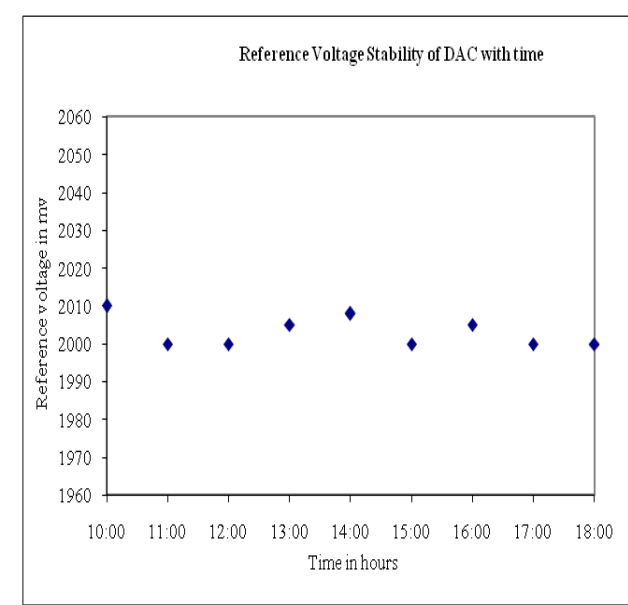

Figure 3a: Stability of Reference Voltage of DAC with load and time 


\section{International Journal of Science and Research (IJSR) \\ ISSN (Online): 2319-7064}

Index Copernicus Value (2013): 6.14 | Impact Factor (2015): 6.391

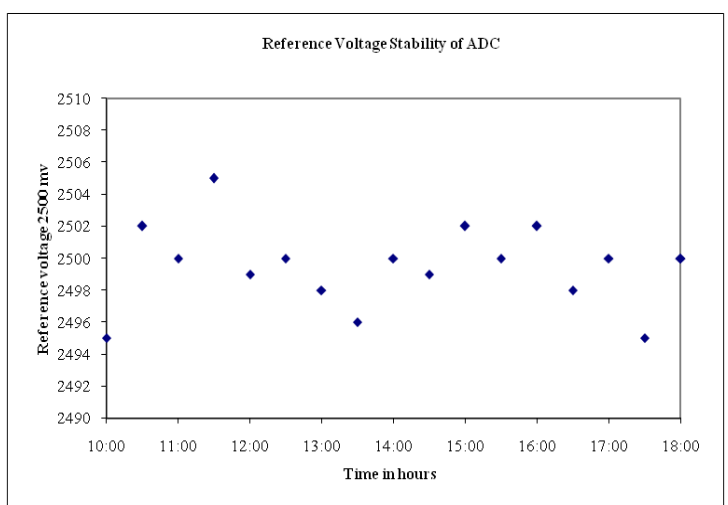

Figure 3b: Stability of Reference Voltage of ADC with time

A typical linearity between the computed voltage and measured voltage is shown in Fig. 4.

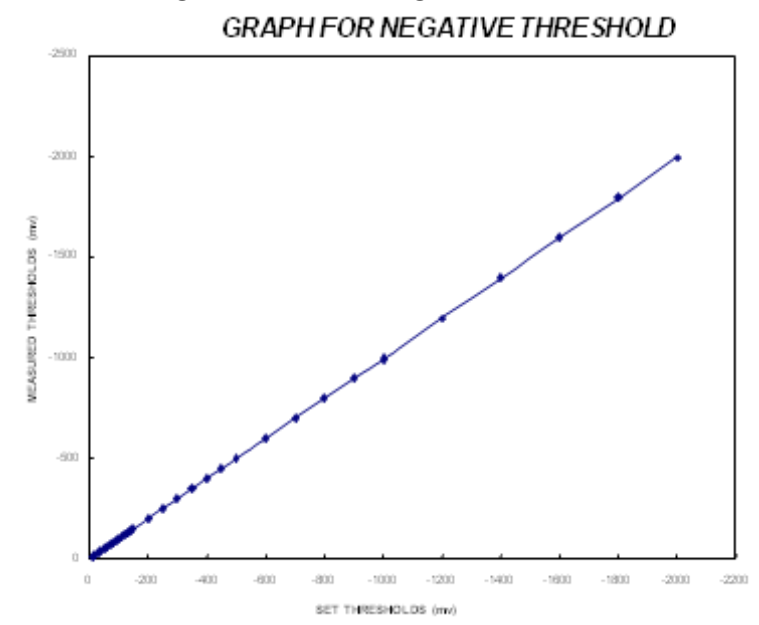

\section{Performance}

The propagation delay of each channel is found to be less than $5 \mathrm{~ns}$ and the rise time \& fall time of less than $1.5 \mathrm{~ns}$ are achieved. The cosmic-ray count rate is monitored by counting pulses from a photomultiplier tube placed on a plastic scintillator block in a dark box using all the discriminators simultaneously along with other commercial available discriminators.

The discriminator threshold voltage is varied in steps and PMT count rates are recorded for each case. The corresponding plots of rate Vs threshold show the expected behavior and are shown in Fig. 6. The graph is matching with the standard commercial module.

Figure 4: Set versus measured voltage

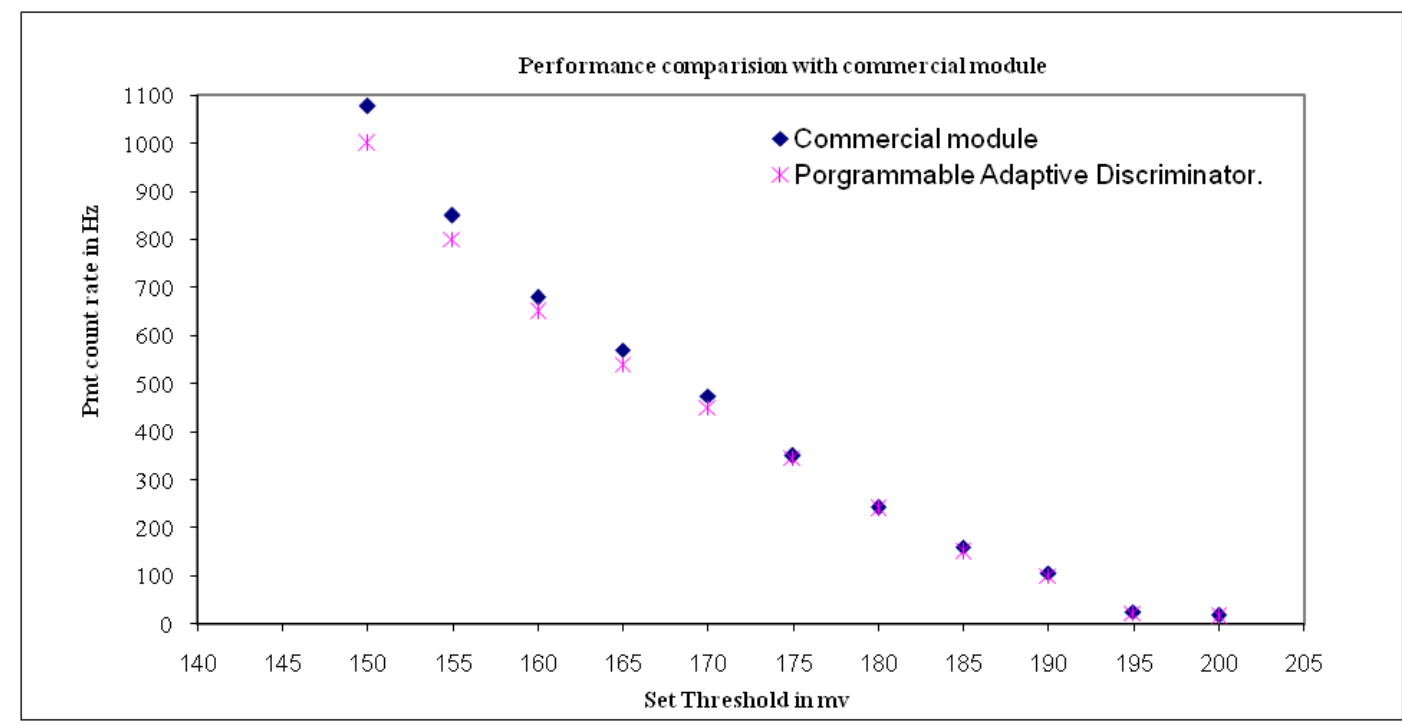

Figure 6: Cosmic ray rate versus threshold voltage

\section{Conclusions}

A prototype of Eight Channel Adaptive Discriminator is fabricated and tested for its functionality. The performance of all the channels of the module is consistent and satisfactory. The chief advantage of this module is that it is of low cost and the threshold voltages of individual channels could be set independently and remotely along with real time monitoring and control features, the rise time and fall time of the output pulse are well within the acceptable range.

\section{Acknowledgements}

I am grateful to the Tata Institute of Fundamental Research (TIFR), Mumbai for providing me with all the facilities required for carrying out this research work and also allowed me to work on the above project. I am very much thankful to

\section{Volume 5 Issue 8, August 2016}




\section{International Journal of Science and Research (IJSR) \\ ISSN (Online): 2319-7064}

Index Copernicus Value (2013): 6.14 | Impact Factor (2015): 6.391

the scientists \& technical members associated with the HEGRO \& HAGAR experimental sites of TIFR. I am very much thankful to Prof. S. C. Choube, Dean, Electrical and Electronics, Rajiv Gandhi Proudyogiki Vishwavidyalaya (RGPV) Bhopal, State Technical University of Madhya Pradesh, Bhopal for his continuous guidance and technical advises from time to time.

\section{References}

[1] V.R.Chitnis et al. ,Status of HAGAR, the high altitude gamma ray observatory at Hanlee. Proc 29th ICRC, Pune, OG 2.7, Vol. 5 (2005), PP 235.

[2] Bhat P.N. et al. „Pachmarhi Array of Cerenkov Telescopes ${ }^{e e}$. Bulletin of Astronomical Society of India (2000), 28, PP 455-457

[3] B.B. Singh et al. "Search for TeV gamma-rays from Geminga pulsar.” Elsevier Astroparticle Physics, vol 23, 2009, p 120.

[4] P. Manjumdar et al. "Pachmarhi Array of Cerenkov Telescopes." Proceedings of International Symposium "The Universe viewed in Gamma-rays", University of Tokyo Symposium, Universal Academy Press 2003, p223.

[5] Upadhya S. S. et al. ,Data Acquisition System for PACT $^{e e}$. Bulletin of ASI 30, (2002), PP 411-416.

[6] Bhat P.N. et al. „Distributed Data Acquisition System for Pachmarhi Array of Cerenkov Telescopes (PACT) ${ }^{\mathrm{ee}}$. Proceeding $27^{\text {th }}$ ICRC, Hamburg, Vol. OG 2.05, (2001), PP 2852.

[7] Lecroy Instrumentation Manual.

[8] IEEE website.

[9] S.K.Gupta et al. ,A high-performance, low cost, leading edge discriminatore. Pramana, Journal of Physics, Indian Academy of Sciences Vol. 65, No.2, August 2005, PP 273-283.

[10] S.K. Sharma et al. "CAMAC based 8 Channel Programmable Discriminator." Journal of Electronics and Telecommunication, Institution of Engineers (India), vol. 90, 2010, p 13. Now it is IEI- Springer Journal series B.

[11] https://www.maximintegrated.com

[12] www.onsemi.com

[13] www.analog.com 